\title{
On baryon properties from a covariant Faddeev approach
}

Helios Sanchis-Alepuz*,

Institut für Physik, Karl-Franzens-Universität Graz, Austria

E-mail: helios.sanchis-alepuz@uni-graz.at

\section{Reinhard Alkofer,}

Institut für Physik, Karl-Franzens-Universität Graz, Austria

\section{Gernot Eichmann,}

Institut für Kernphysik, Technische Universität Darmstadt, Germany

\section{Selym Villalba-Chávez,}

Institut für Physik, Karl-Franzens-Universität Graz, Austria

The Poincaré-covariant Faddeev equation and its solution in rainbow-ladder truncation, i.e., with a generalized gluon exchange as irreducible two-particle-interaction, is presented. The covariant decomposition of baryon amplitudes, representing relativistic three-quark states, is discussed and explicitly given for the $\Delta$-baryon. The calculated $\Delta$ mass compares favourably with the experimental one.

Light Cone 2010: Relativistic Hadronic and Particle Physics

June 14-18, 2010

Valencia, Spain

\footnotetext{
${ }^{*}$ Speaker.
} 


\section{Introduction}

It is generally accepted that QCD is the theory of strong interactions and thus hadrons are bound states of quarks and glue. Any numerical solution of QCD's bound state equations, i.e. the Bethe-Salpeter equation for mesons and the Faddeev equation for baryons, requires Green functions of quarks and gluons as input. To this end it is interesting to note that in Landau gauge the quark and gluon propagators are sufficiently well known for phenomenological purposes; for recent reviews see, e.g., $[1,2]$. The aim is, of course, to use these results, in particular the ones for the quark correlations, in the relativistic bound state equations for baryons in a similar manner as for mesons. However, the relativistic bound-state description for baryons is considerably less understood than for mesons, even on the phenomenological level. Therefore the complicated task of implementing our knowledge of the strong-interaction domain of QCD in a first-principle continuum description of baryons as Poincaré-covariant three-quark bound states is only approached in small steps.

Over the last decade one has mostly followed an approach starting from a covariant Faddeev equation [3] but immediately reducing its complexity by treating the two-body $T$-matrix in a separable expansion. Due to the importance of quark-quark correlations, so-called diquarks, this approximative treatment has been called quark-diquark approach [4-7]. Reducing the Faddeev equation to an effective two-body equation allowed for the calculation of many different baryon observables; for a collection of recent results see, e.g., [8-12].

Only recently it was possible to solve the full Poincaré-covariant Faddeev equation for the nucleon [13-15]. Its evolution with the pion mass reveals already quite interesting features, especially when compared to corresponding lattice results. It still remains a challenging task to compute observables other than the mass of the nucleon.

On the other hand, the lowest lying excited state of the nucleon, the $\Delta(1232)$ resonance, enjoys a special place in the family of baryon resonances. The $\Delta(1232)$ resonance has the highest production cross section of all nucleon resonances, and it is a spin $3 / 2$ particle with the same quark content as the nucleon. Therefore, it seems obvious to attempt also for the $\Delta(1232)$ the corresponding solution of the Poincaré-covariant Faddeev equation. In the following we will report on progress in this direction by discussing the construction of the $\Delta$ 's Faddeev amplitude and by giving a (first) result for its mass.

\section{Faddeev equation}

Baryons in QCD appear as poles in the three-quark scattering matrix. Analyzing the residue at the pole associated with a baryon of mass $M$ allows to derive a relativistic equation for this bound state:

$$
\boldsymbol{\Psi}=\boldsymbol{K}_{3} \boldsymbol{\Psi}, \quad \boldsymbol{K}_{3}=\boldsymbol{K}_{3}^{i r r}+\sum_{a=1}^{3} \boldsymbol{K}_{(2)}^{(a)},
$$

where now the baryon is described on-shell by the bound-state amplitude $\boldsymbol{\Psi}$. This equation includes all possible correlations among the three quarks, which comprises a three-body irreducible contribution as well as the sum of the three possible two-body irreducible interactions with a spectator quark, denoted by $a$. 


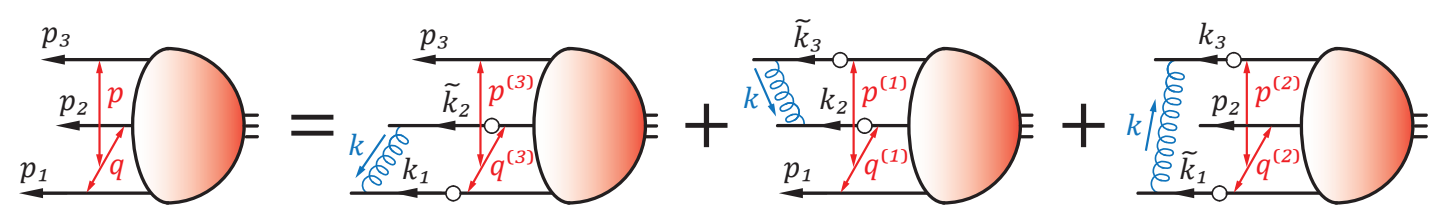

Figure 1: Diagrammatical representation of the Faddeev equation in rainbow-ladder truncation

The success of the quark-diquark approach to baryon properties supports the idea that the quark-quark correlations dominate in binding baryons. Omitting the three-body irreducible contribution leads to the covariant Faddeev equation [3]

$$
\boldsymbol{\Psi}_{\alpha \beta \gamma J}(p, q, P)=\sum_{a=1}^{3} \int_{k} \boldsymbol{K}_{\alpha \alpha^{\prime} \beta \beta^{\prime} \gamma \gamma^{\prime}}^{(a)} \boldsymbol{\Psi}_{\alpha^{\prime} \beta^{\prime} \gamma^{\prime} J}\left(p^{(a)}, q^{(a)}, P\right),
$$

where $\{\alpha, \beta, \gamma\}$ are Dirac indices for the three valence quarks and the nature of the fourth index $J$ depends on the baryon of interest. The momentum dependence of the amplitude on the three quark momenta $p_{1}, p_{2}, p_{3}$ is here reexpressed in terms of the total momentum $P$ and two relative momenta $p$ and $q$, where $P^{2}=-M^{2}$ is fixed. They are related via:

$$
\begin{array}{ccc}
p=(1-\zeta) p_{3}-\zeta p_{d}, & q=\frac{p_{2}-p_{1}}{2}, & P=p_{1}+p_{2}+p_{3}, \\
p_{1}=-q-\frac{p}{2}+\frac{1-\zeta}{2} P, & p_{2}=q-\frac{p}{2}+\frac{1-\zeta}{2} P, & p_{3}=p+\zeta P,
\end{array}
$$

where we abbreviated $p_{d}:=p_{1}+p_{2}$. We use the value $\zeta=1 / 3$ which maximizes the upper boundary for the nucleon mass with respect to restrictions arising from the quark propagator's singularity structure; for a detailed description see, e.g., refs. $[14,16]$. The internal relative momenta are given by:

$$
\begin{array}{ccc}
p^{(1)}=p+k, & p^{(2)}=p-k, & p^{(3)}=p, \\
q^{(1)}=q-k / 2, & q^{(2)}=q-k / 2, & q^{(3)}=q+k .
\end{array}
$$

Furthermore, $\boldsymbol{K}^{(a)}$ denotes the renormalization-group invariant products of a $q q$ kernel and two dressed quark propagators:

$$
\begin{aligned}
& \boldsymbol{K}_{\alpha \alpha^{\prime} \beta \beta^{\prime} \gamma \gamma^{\prime}}^{(1)}=\delta_{\alpha \alpha^{\prime}} \mathcal{K}_{\beta \beta^{\prime \prime} \gamma \gamma^{\prime \prime}} S_{\beta^{\prime \prime} \beta^{\prime}}\left(k_{2}\right) S_{\gamma^{\prime \prime} \gamma^{\prime}}\left(\widetilde{k}_{3}\right), \\
& \boldsymbol{K}_{\alpha \alpha^{\prime} \beta \beta^{\prime} \gamma \gamma^{\prime}}^{(2)}=\delta_{\beta \beta^{\prime}} \mathcal{K}_{\gamma \gamma^{\prime \prime} \alpha \alpha^{\prime \prime}} S_{\gamma^{\prime \prime} \gamma^{\prime}}\left(k_{3}\right) S_{\alpha^{\prime \prime} \alpha^{\prime}}\left(\widetilde{k}_{1}\right), \\
& \boldsymbol{K}_{\alpha \alpha^{\prime} \beta \beta^{\prime} \gamma \gamma^{\prime}}^{(3)}=\delta_{\gamma \gamma^{\prime}} \mathcal{K}_{\alpha \alpha^{\prime \prime} \beta \beta^{\prime \prime}} S_{\alpha^{\prime \prime} \alpha^{\prime}}\left(k_{1}\right) S_{\beta^{\prime \prime} \beta^{\prime}}\left(\widetilde{k}_{2}\right) .
\end{aligned}
$$

The quark propagators $S$ involved in these expressions depend on the internal quark momenta $k_{i}=p_{i}-k$ and $\widetilde{k}_{j}=p_{j}+k$, where $k$ is the gluon momentum. 


\section{Rainbow-ladder truncation}

In order to perform our calculation we have to specify the $q q$ kernel as well as the dressed propagators. We require our kernel to preserve the axial-vector Ward-Takahashi identity, so that chiral symmetry and its dynamical breaking is correctly implemented. The simplest realization of such a symmetry-preserving truncation is the rainbow-ladder truncation; for its definition within relativistic three-body equations see refs. $[14,17]$. It describes the $q q$ kernel by a ladder dressedgluon exchange

$$
\mathcal{K}_{\alpha \alpha^{\prime} \beta \beta^{\prime}}(k)=Z_{2}^{2} \frac{4 \pi \alpha\left(k^{2}\right)}{k^{2}} T_{k}^{\mu \nu} \gamma_{\alpha \alpha^{\prime}}^{\mu} \gamma_{\beta \beta^{\prime}}^{\nu}
$$

which is also involved in the Dyson-Schwinger equation (for reviews see e.g., $[1,18,19]$ ) for the renormalized dressed quark propagator:

$$
S_{\alpha \beta}^{-1}(p)=Z_{2}(i p+m)_{\alpha \beta}+\int_{q} \mathcal{K}_{\alpha \alpha^{\prime} \beta^{\prime} \beta}(k) S_{\alpha^{\prime} \beta^{\prime}}(q)
$$

Here the bare quark mass $m$ enters as an input and $k=p-q$. The color structure of the kernel generates the prefactors $2 / 3$ and $4 / 3$ for the integrals in Eq. (2.2) and Eq. (3.2), respectively. Eq. (3.1) involves an effective coupling $\alpha\left(k^{2}\right)$ for which we will henceforth use the parametrization given in [20]:

$$
\alpha\left(k^{2}\right)=\pi \eta^{7}\left(\frac{k^{2}}{\Lambda^{2}}\right)^{2} e^{-\eta^{2}\left(\frac{k^{2}}{\Lambda^{2}}\right)}+\alpha_{U V}\left(k^{2}\right) .
$$

While the first term describes the non-perturbative enhancement at small and intermediate gluon momenta necessary to generate dynamical chiral symmetry breaking and the dynamical generation of a constituent-quark mass scale, the second term reproduces the one-loop QCD perturbative running coupling $\alpha_{U V}\left(k^{2}\right)$ at large gluon momentum $\left(k^{2} \gg \Lambda_{Q C D}^{2}\right)$. The infrared behavior is parameterized by the dimensionless parameter $\eta$ and the infrared scale $\Lambda$. Hereafter we will fix the latter to $\Lambda=0.72 \mathrm{GeV}$ which has been used in $[8,20,21]$ to reproduce experimental results for meson and baryon properties. We remark that ground-state properties in this framework have turned out to be insensitive to the choice of $\eta$ in a certain range [21].

\section{Covariant decomposition of baryon amplitudes}

Numerical assessment of the amplitude and mass of a particular baryon requires to decompose the Faddeev amplitude in a suitable basis. This can be done independently of any approximation but based solely on first principles like Poincaré covariance, spatial and time-reversal symmetries and charge-conjugation properties. Considering this idea, a convenient basis for the nucleon amplitude was obtained in Refs. [13-15]. The latter turns out to be a four-rank tensor $\boldsymbol{\Psi}_{\alpha \beta \gamma \delta}($ i.e., $J \equiv \delta$ ) in Dirac space whose dimension is 256 . However, for a nucleon of positive parity and positive energy its amplitude can be expanded in terms of a complete orthogonal basis which only involves 64 Dirac structures:

$$
\boldsymbol{\Psi}_{\alpha \beta \gamma \delta}=\sum_{\ell=1}^{64} \varkappa_{\ell} \boldsymbol{\Phi}_{\alpha \beta \gamma \delta}^{(\ell)} .
$$


Each dressing function $\varkappa_{\ell}$ in Eq. (4.1) is a dimensionless scalar factor which depends on five Lorentz-invariant combinations:

$$
p^{2}, \quad q^{2}, \quad z_{0}=\widehat{p_{T}} \cdot \widehat{q_{T}}, \quad z_{1}=\hat{p} \cdot \hat{P}, \quad z_{2}=\hat{q} \cdot \hat{P} .
$$

In our framework a hat denotes a normalized four-vector ${ }^{1}$, and $p_{T}^{\mu}=T_{P}^{\mu v} p^{v}$ with $T_{P}^{\mu v}=\delta^{\mu v}-\hat{P}^{\mu} \hat{P}^{v}$ is a transverse projector with respect to the baryon momentum.

It is noteworthy that the basis $\{\boldsymbol{\Phi}\}$ can be divided into two sets $\left\{\mathcal{S}_{i j}^{r}, \mathcal{P}_{i j}^{r}\right\}$ whose elements

$$
\mathcal{S}_{i j}^{r}=(\mathbb{I} \otimes \mathbb{I})\left(\Gamma_{i} \otimes \Gamma_{j}\right) \Omega_{r}(\hat{P}) \quad \text { and } \quad \mathcal{P}_{i j}^{r}=\left(\gamma^{5} \otimes \gamma^{5}\right)\left(\Gamma_{i} \otimes \Gamma_{j}\right) \Omega_{r}(\hat{P}),
$$

with

$$
\Omega_{r}(\hat{P})=\Lambda_{r}(\hat{P}) \gamma^{5} C \otimes \Lambda_{+}(\hat{P}),
$$

involve the charge conjugation-matrix $C=\gamma^{4} \gamma^{2}$, the positive- and negative-energy projectors $\Lambda_{ \pm}(\hat{P})=$ $(\mathbb{I} \pm \hat{P}) / 2$, and the elements encoded in

$$
\Gamma_{i}=\left\{\mathbb{I}, \frac{1}{2}\left[\widehat{p_{T}}, \widehat{q_{t}}\right], \widehat{p_{T}}, \widehat{q_{t}}\right\}
$$

where $q_{t}^{\mu}=T_{p_{T}}^{\mu \lambda} T_{P}^{\lambda v} q^{v}=T_{p_{T}}^{\mu \lambda} q_{T}^{\lambda}$. Note that the expression above introduces a relative-momentum dependence in our amplitude. According to this notation, a particular basis element with index $\ell$ is fully determined by a combination of indices $i, j, r$ with $r= \pm$ and $i, j=1, \ldots, 4$.

In contrast to the previous case, the covariant $\Delta$-amplitude is a mixed tensor in which five indices are involved. Four of them are spinorial whereas the remaining one is a four-vector index. As a consequence the amplitude can be written as

$$
\boldsymbol{\Psi}_{\alpha \beta \gamma \delta}^{\mu}=\sum_{\ell} \varkappa_{\ell} \boldsymbol{\Phi}_{\alpha \beta \gamma \delta}^{(\ell) \mu}
$$

The spin-3/2 value of the $\Delta$ is then obtained by applying the Rarita-Schwinger projector

$$
\mathbb{P}_{+}^{\mu v}(\hat{P})=\Lambda_{+}(\hat{P})\left(T_{P}^{\mu v}-\frac{1}{3} \gamma_{T}^{\mu} \gamma_{T}^{v}\right), \quad \gamma_{T}^{\mu}=T_{P}^{\mu v} \gamma^{v},
$$

which is restricted to positive energy and obeys the constrains $\hat{P}^{\mu} \mathbb{P}_{+}^{\mu v}(\hat{P})=\gamma_{T}^{\mu} \mathbb{P}_{+}^{\mu v}(\hat{P})=0$.

Now, in order to construct the basis for Eq. (4.5), we exploit the 64 covariant structures given in Eq. (4.2) and proceed by multiplying $\mathcal{S}_{i j}^{r}$ and $\mathcal{P}_{i j}^{r}$ with all possible positive-parity combinations

$$
\begin{array}{lllll}
\gamma_{T}^{\mu} \gamma^{5} \otimes \mathbb{I}, & \gamma^{5} \otimes \gamma_{T}^{\mu}, & \widehat{p}_{T}^{\mu} \gamma^{5} \otimes \mathbb{I}, & \widehat{q}_{t}^{\mu} \gamma^{5} \otimes \mathbb{I}, & \hat{P}^{\mu} \gamma^{5} \otimes \mathbb{I}, \\
\mathbb{I} \otimes \gamma_{T}^{\mu} \gamma^{5}, & \gamma_{T}^{\mu} \otimes \gamma^{5}, & \mathbb{I} \otimes \widehat{p}_{T}^{\mu} \gamma^{5}, & \mathbb{I} \otimes \widehat{q}_{t}^{\mu} \gamma^{5}, & \mathbb{I} \otimes \hat{P}^{\mu} \gamma^{5}
\end{array}
$$

This exercise generates 640 preliminary covariant structures which overcount the actual number of independent basis elements. Indeed, because of the following identities

$$
\begin{aligned}
\left(\gamma_{T}^{\mu} \otimes \gamma^{5}\right)\left(\begin{array}{c}
\mathcal{S}_{i j}^{r} \\
\mathcal{P}_{i j}^{r}
\end{array}\right)= & \left(\gamma_{T}^{\mu} \gamma^{5} \otimes \mathbb{I}\right)\left(\begin{array}{c}
\mathcal{P}_{i j}^{r} \\
\mathcal{S}_{i j}^{r}
\end{array}\right), \quad\left(\mathbb{I} \otimes \gamma^{5}\right)\left(\begin{array}{c}
\mathcal{S}_{i j}^{r} \\
\mathcal{P}_{i j}^{r}
\end{array}\right)=\left(\gamma^{5} \otimes \mathbb{I}\right)\left(\begin{array}{c}
\mathcal{P}_{i j}^{r} \\
\mathcal{S}_{i j}^{r}
\end{array}\right), \\
& \left(\gamma^{5} \otimes \gamma_{T}^{\mu}\right)\left(\begin{array}{c}
\mathcal{S}_{i j}^{r} \\
\mathcal{P}_{i j}^{r}
\end{array}\right)=\left(\mathbb{I} \otimes \gamma_{T}^{\mu} \gamma^{5}\right)\left(\begin{array}{c}
\mathcal{S}_{i j}^{r} \\
\mathcal{P}_{i j}^{r}
\end{array}\right)
\end{aligned}
$$

\footnotetext{
${ }^{1}$ We work in Euclidean space.
} 
we can restrict ourselves to the upper row in Eq. (4.6) and halve the number given above to 320. A further reduction is achieved by contracting the former elements with $\mathbb{I} \otimes \mathbb{P}_{+}^{\mu v}$ which projects out the terms involving $\hat{P}^{\mu}$. Consequently, 320-64 = 256 covariant structures remain. The 64 elements generated from $\left(\gamma^{5} \otimes \gamma_{T}^{\mu}\right)$ can be ruled out as well since they turn out to be linear combinations of the remaining $256-64=192$ basis elements:

\begin{tabular}{|c||c||c|}
\hline$\left[\mathscr{G}_{\mathrm{g}}\right]_{i j}^{r}$ & {$\left[\mathscr{P}_{\mathrm{g}}\right]_{i j}^{r}$} & {$\left[\mathscr{Q}_{\mathrm{g}}\right]_{i j}^{r}$} \\
\hline \hline$\left(\gamma^{\mu} \gamma_{5} \otimes \mathbb{I}\right)\left(\begin{array}{c}\mathcal{S}_{i j}^{r} \\
\mathcal{P}_{i j}^{r}\end{array}\right)\left(\mathbb{I} \otimes \mathbb{P}_{+}^{\mu v}\right)$ & $\left(\gamma_{5} \otimes \mathbb{I}\right)\left(\begin{array}{c}\mathcal{S}_{i j}^{r} \\
\mathcal{P}_{i j}^{r}\end{array}\right)\left(\mathbb{I} \otimes{\widehat{p_{T}}}^{\mu} \mathbb{P}_{+}^{\mu v}\right)$ & $\left(\gamma_{5} \otimes \mathbb{I}\right)\left(\begin{array}{c}\mathcal{S}_{i j}^{r} \\
\mathcal{P}_{i j}^{r}\end{array}\right)\left(\mathbb{I} \otimes \widehat{q}_{t}^{\mu} \mathbb{P}_{+}^{\mu v}\right)$ \\
\hline
\end{tabular}

in which $\mathfrak{g}=\{\mathcal{S}, \mathcal{P}\}$. Only 128 of them are linearly independent. We are free to choose a linearly independent subset; in particular, the following choice is orthonormal:

$$
\begin{aligned}
& \sqrt{\frac{3}{2}}\left[\mathscr{P}_{\mathrm{g}}\right]_{i j}^{r} \\
& \sqrt{2}\left(\left[\mathscr{Q}_{\mathfrak{g}}\right]_{i 1}^{r}-\frac{1}{2}\left[\mathscr{P}_{\mathrm{g}}\right]_{i 2}^{r}\right), \quad \sqrt{2}\left(\left[\mathscr{Q}_{\mathrm{g}}\right]_{i 2}^{r}+\frac{1}{2}\left[\mathscr{P}_{\mathrm{g}}\right]_{i 1}^{r}\right), \\
& \sqrt{2}\left(\left[\mathscr{Q}_{\mathfrak{g}}\right]_{i 3}^{r}-\frac{1}{2}\left[\mathscr{P}_{\mathfrak{g}}\right]_{i 4}^{r}\right), \quad \sqrt{2}\left(\left[\mathscr{Q}_{\mathfrak{g}}\right]_{i 4}^{r}+\frac{1}{2}\left[\mathscr{P}_{\mathfrak{g}}\right]_{i 3}^{r}\right)
\end{aligned}
$$

We note that it is rather complicated to perform a partial-wave decomposition, $c f$. [16], for this basis. A better procedure would be to apply a Gram-Schmidt algorithm and include the elements $\left[\mathscr{G}_{\mathfrak{g}}\right]_{11}^{r}$ which are likely to be the dominant contributions. Such an analysis is beyond the scope of the present manuscript but a study in this direction is in progress.

\section{Results and outlook}

Having defined an orthonormal basis for the $\Delta$-baryon amplitude, the numerical method described in $[14,15]$ can be directly applied. The solution must have the correct symmetry under the permutation group. Namely, both the flavour and the Poincaré-Dirac parts of the amplitudes have to be fully symmetric under the exchange of any two quarks, as required by the Pauli principle. Thanks to the iterative procedure used to solve the equations, to fulfill this condition we just use $\left[\mathscr{G}_{\mathfrak{g}}\right]_{11}^{+}$, which has the correct symmetry, as the starting function. This element does not appear explicitly in the basis (4.8) but can, of course, be expressed as a linear combination of the given basis elements.

The resulting $\Delta$ mass at the physical pion mass is given in Table 1 . We see that the mass is slightly overestimated with respect to the physical value, indicating that further structures need to be taken into account. Corrections to this value, though small, would arise from considering kernels truncated beyond rainbow-ladder and also including the 3-body irreducible contributions in the bound-state equation. On the other hand, the mass in this approach is only minimally below the quark-diquark result [10] in which only the axial-vector diquark was considered, which means that the diquark approximation works already very well. 


\begin{tabular}{|l||c||c|}
\hline$M_{\Delta}^{\text {phys }}$ & $M_{\Delta}^{Q-D Q}$ & $M_{\Delta}^{\text {Faddeev }}$ \\
\hline 1.23 & 1.28 & 1.26 \\
\hline
\end{tabular}

Table 1: $\Delta$ mass (in $\mathrm{GeV}$ ) obtained from the Faddeev equation compared to the physical value and to the quark-diquark calculation.

The results presented here are, however, the first piece in a more elaborated investigation. First of all, as said above, it would be convenient to have a partial wave decomposition of the basis to gain physical insight and ease comparison with quark model calculations. Also, the dependence of our results with the parameters in the effective coupling must be analyzed. Finally, the ingredients needed to calculate the $\Delta$-baryon mass and structure allow us to perform the same study for the $\Omega$-baryon, with the only difference being the quark mass and the corresponding propagator. To compare with lattice data a calculation for a range of quark masses is anyhow desirable. Work in these directions is in progress.

\section{Acknowledgments}

H.S.-A. thanks the organizers of this highly interesting workshop for the possibility to present his recent work. We are grateful to M. Blank, C.S. Fischer, A. Krassnigg, D. Nicmorus and R. Williams for helpful discussions. This work was supported by the Austrian Science Fund FWF under Project No. P20592-N16, Erwin-Schrödinger Fellowship J3039-N16, and the Doctoral Program W1203; as well as the Helmholtz Young Investigator Grant VH-NG-332 and in part by the European Union (HadronPhysics2 project "Study of strongly-interacting matter").

\section{References}

[1] C. S. Fischer, J. Phys. G 32 (2006) R253 [arXiv:hep-ph/0605173].

[2] C. S. Fischer, A. Maas and J. M. Pawlowski, Annals Phys. 324 (2009) 2408 [arXiv:0810.1987 [hep-ph]].

[3] L. D. Faddeev, Sov. Phys. JETP 12 (1961) 1014 [Zh. Eksp. Teor. Fiz. 39 (1960) 1459].

[4] G. Hellstern et al., Nucl. Phys. A 627 (1997) 679 [arXiv:hep-ph/9705267].

[5] M. Oettel et al., Phys. Rev. C 58 (1998) 2459 [arXiv:nucl-th/9805054].

[6] J. C. R. Bloch et al., Phys. Rev. C 60 (1999) 062201 [arXiv:nucl-th/9907120].

[7] M. Oettel, R. Alkofer, and L. von Smekal, Eur. Phys. J. A8 (2000) 553-566 [arXiv:nucl-th/0006082].

[8] G. Eichmann et al., Annals Phys. 323 (2008) 2505 [arXiv:0712.2666 [hep-ph]].

[9] G. Eichmann et al., Phys. Rev. C 77 (2008) 042202 [arXiv:0802.1948 [nucl-th]]; Phys. Rev. C 79 (2009) 012202 [arXiv:0810.1222 [nucl-th]]. 
[10] D. Nicmorus et al., Phys. Rev. D 80 (2009) 054028 [arXiv:0812.1665 [hep-ph]].

[11] D. Nicmorus, G. Eichmann and R. Alkofer, arXiv:1008.3184 [hep-ph].

[12] G. Eichmann et al., arXiv:1010.0206 [hep-ph], D. Nicmorus et al., arXiv:1008.4149 [hep-ph],

[13] G. Eichmann et al., Phys. Rev. Lett. 104 (2010) 201601 [arXiv:0912.2246 [hep-ph]].

[14] G. Eichmann, PhD Thesis, Graz University (2009) [arXiv:0909.0703 [hep-ph]].

[15] G. Eichmann et al., EPJ Web Conf. 3 (2010) 03028 [arXiv:0912.2876 [hep-ph]], R. Alkofer et al., Chin. Phys. C 34 (2010) 1175 [arXiv:0912.3105 [hep-ph]].

[16] M. Oettel, PhD Thesis, Tübingen University (2000) [arXiv:nucl-th/0012067].

[17] J. G. Taylor, Phys. Rev. 150 (1966) 1321.

[18] C. D. Roberts and S. M. Schmidt, Prog. Part. Nucl. Phys. 45 (2000) S1 [arXiv:nucl-th/0005064].

[19] R. Alkofer and L. von Smekal, Phys. Rept. 353 (2001) 281 [arXiv:hep-ph/0007355].

[20] P. Maris and P. C. Tandy, Phys. Rev. C 60 (1999) 055214 [arXiv:nucl-th/9905056].

[21] A. Krassnigg, Phys. Rev. D 80 (2009) 114010 [arXiv:0909.4016 [hep-ph]]. 